La Revue

des Droits

de l'Homme

\section{La Revue des droits de l'homme}

Revue du Centre de recherches et d'études sur les droits fondamentaux

Actualités Droits-Libertés | 2015

\title{
De l'éloignement à la neutralisation : la CJUE navigue à vue dans la mer de l'irrégularité
}

Droits des étrangers (Directive 2008/115/CE)

\section{Chloé Peyronnet}

\section{OpenEdition}

\section{Journals}

Édition électronique

URL : https://journals.openedition.org/revdh/1502

DOI : $10.4000 /$ revdh. 1502

ISSN : 2264-119X

Éditeur

Centre de recherches et d'études sur les droits fondamentaux

Référence électronique

Chloé Peyronnet, « De l'éloignement à la neutralisation : la CJUE navigue à vue dans la mer de l'irrégularité », La Revue des droits de l'homme [En ligne], Actualités Droits-Libertés, mis en ligne le 28 octobre 2015, consulté le 25 janvier 2022. URL : http://journals.openedition.org/revdh/1502 ; DOI : https://doi.org/10.4000/revdh.1502

Ce document a été généré automatiquement le 25 janvier 2022.

Tous droits réservés 


\section{De l'éloignement à la neutralisation : la CJUE navigue à vue dans la mer de l'irrégularité}

Droits des étrangers (Directive 2008/115/CE)

\section{Chloé Peyronnet}

1 En posant une question préjudicielle en interprétation à la Cour de justice dans l'affaire Celaj, le tribunal de Florence lui donnait l'occasion de préciser ses jurisprudences El Dridi $^{1}$, Achughbabian ${ }^{2}$ et $\mathrm{Md}$ Sagor $^{3}$ relatives à l'articulation de la 2008/115/CE (directive "retour») avec la compétence pénale des États membres". Elle y était d'ailleurs expressément invitée par l'avocat général Maciej Szpunar ${ }^{5}$, dont elle a cependant ignoré les conclusions éclairées.

2 Ressortissant albanais, Skerdjan Celaj avait été condamné pénalement pour un vol à l'arraché en Italie, où il résidait sans statut légal. Après avoir exécuté sa peine, il avait fait l'objet d'une décision de retour et d'une interdiction d'entrée de trois ans à compter du 17 avril 2012. Bien que la procédure de retour n'ait pas abouti pour des raisons techniques, Skerdjan Celaj avait finalement quitté de lui-même le territoire italien, sur lequel il est toutefois revenu avant l'échéance de son interdiction d'entrée. Il a alors été arrêté et poursuivi pénalement pour infraction à l'article $13 \$ 13 \mathrm{du}$ décret législatif $n^{\circ} 286 / 1998^{6}$, qui punit d'une peine d'emprisonnement de un à quatre ans le fait de pénétrer sur le territoire national sans autorisation ministérielle après avoir fait l'objet d'une décision de retour.

3 Saisi de cette affaire, le tribunal de Florence a dû apprécier la conformité dudit décret législatif à la directive retour. En effet, au regard des décisions de la Cour de justice enjoignant aux États membres de respecter la "gradation des mesures coercitives" prévues par la directive retour et le principe de proportionnalité ${ }^{7}$, le défendeur a cru pouvoir contester le fait qu'une peine privative de liberté lui soit applicable sans qu'un départ volontaire, une alternative à la rétention ou un placement en rétention à des fins d'éloignement n'ait prouvé son inefficacité. En cela, il mobilisait de manière pertinente la jurisprudence de la Cour de justice ${ }^{8}$ que l'avocat général a résumé ainsi : 
«il n'y a pas de place pour une [peine privative de liberté] si l'objectif de la directive est de prévoir le retour rapide de ressortissants de pays tiers en séjour irrégulier " .

4 Cependant, bien que la Cour de justice tente de l'inscrire dans la continuité de sa jurisprudence, l'arrêt Celaj s'avère en réalité peu conforme aux principes appliqués précédemment quant à l'articulation de la directive retour avec la compétence pénale des États membres. Ainsi, balayant le principe de coopération loyale pourtant largement invoqué auparavant, elle semble faire fi de l'argument de «l'effet utile » qui fondait ses décisions antérieures. À y bien regarder, elle s'attache ici à remodeler l'objectif de la directive retour $\left(1^{\circ}\right)$ : son effet utile n'impliquerait pas tant la facilitation de l'éloignement que la neutralisation des ressortissants de pays tiers en situation irrégulière $\left(2^{\circ}\right)$, dont le retour et la condamnation à des peines privatives de liberté seraient deux variantes interchangeables. Lucides quant à la crispation des souverainetés nationales et timides face à la problématique des migrants nonéloignables, les juges de Luxembourg paraissent naviguer à vue, jonglant entre considérations politiques et principes juridiques au détriment tant de l'effet utile de la directive que des droits fondamentaux.

\section{$1 \%$}

\section{La distorsion de l'argument de l'effet utile : Un remodèlement de l'objectif de la directive retour}

5 Jusqu'alors, l'argument de l'effet utile de la directive retour était invoqué par la Cour de justice pour encadrer la compétence pénale des États membres. Souhaitant à présent leur donner une plus grande latitude, elle se voit prise à son propre jeu et en rejette les conséquences (A). S'il est silencieux car mal assumé, c'est bien un virage jurisprudentiel qu'opère ici la Cour de justice (B).

\section{A - L'argument de l'effet utile : La Cour de justice prise à son propre jeu}

Conformément aux arrêts El Dridi, Achughbabian et Md Sagor, la Cour de justice rappelle que si ni la directive retour ni sa base juridique ${ }^{10}$ n'ont pour objet d'encadrer la compétence pénale des États membres, ils ne peuvent néanmoins l'exercer que dans la mesure où elle ne compromet pas l'effet utile de ladite directive (\$§ 20-21). C'est en usant de ce raisonnement que la Cour de justice a conduit les États membres à opérer un glissement du droit pénal vers le droit des étrangers.

7 En effet, l'entrée irrégulière est passible d'une peine d'emprisonnement dans dix-sept des vingt-huit États membres de l'Union européenne, tandis que le séjour irrégulier est passible d'emprisonnement dans dix d'entre eux ${ }^{11}$. Or, l'objectif de la directive retour consiste dans "la mise en place d'une politique efficace d'éloignement et de rapatriement fondée sur des normes communes afin que les personnes concernées soient rapatriées d'une façon humaine et dans le respect intégral de leurs droits fondamentaux ainsi que de leur dignité $»^{12}$. De ce fait, la Cour de justice a jusqu'alors considéré que toute peine privative de liberté ralentissait le retour des ressortissants de pays tiers en situation irrégulière en les maintenant sur le territoire national pour tout le temps de leur emprisonnement. 
8 Cette jurisprudence, qui peut de prime abord sembler condamner la «double-peine $»^{13}$, n'était pas tant motivée par le volet «droit fondamentaux » de l'objectif précité que par le souci d'accélérer le transfert physique des intéressés vers le territoire d'État tiers. Ainsi, la Cour de justice réaffirme clairement qu' « il serait porté atteinte aux normes et aux procédures communes établies par la directive 2008/115 si, après avoir constaté le séjour irrégulier d'un ressortissant d'un pays tiers, l'État membre concerné faisait précéder l'exécution de la décision de retour, voire l'adoption même de cette décision, d'une poursuite pénale pouvant conduire à une peine d'emprisonnement au cours de la procédure de retour, dans la mesure où une telle démarche risquerait de retarder l'éloignement» (\$26). C'est ainsi qu'elle a pu considérer l'infliction d'une amende pour séjour irrégulier comme conforme à l'objectif de la directive du fait qu'elle ne ralentissait pas la procédure de retour ${ }^{14}$; de même pour la condamnation à une assignation à résidence pour séjour irrégulier, elle aussi validée par la Cour de justice dans la mesure où elle pouvait être levée dès lors que l'exécution de la décision de retour devenait possible ${ }^{15}$.

Surtout, la Cour de justice permet expressément la condamnation à une peine privative de liberté pour entrée ou séjour irrégulier d'une personne ayant fait l'objet d'une procédure de retour qui, en dépit de l'épuisement des mesures coercitives prévues par la directive retour, s'est avérée infructueuse - peu important que l'échec de la procédure soit lié au comportement de l'intéressé ou à des difficultés administratives indépendantes de sa volonté ${ }^{16}$. Autrement dit, pour les ressortissants de pays tiers en situation irrégulière, décision de retour et prison peuvent se cumuler à condition que cet ordre soit respecté et qu'il ait été recouru à la rétention à des fins d'éloignement laquelle est au sommet de l'échelle des mesures coercitives prévues par la directive retour - avant d'opter pour une privation de liberté de nature pénale.

10 C'est au nom de cette jurisprudence, centrée sur la nécessité d'assurer l'effet utile de la directive retour et l'obligation de coopération loyale des États membres, que l'avocat général demandait à la Cour de justice de juger la réglementation italienne incompatible avec la directive retour ${ }^{17}$. En effet, que l'entrée irrégulière sur le territoire national soit une première ou une seconde occurrence, il n'en reste pas moins qu'une peine privative de liberté maintient le ressortissant de pays tiers concerné sur place et empêche donc son retour pendant toute la durée de son exécution. Pourtant, c'est sans même évoquer les conclusions de son avocat général que la Cour de justice balaie cet argumentaire : refusant d'être prise à son propre jeu, elle a choisi d'opérer un virage jurisprudentiel silencieux dont la nature finaliste n'est que trop manifeste.

\section{$B$ - Le rejet des conséquences de l'effet utile : Un virage jurisprudentiel silencieux}

11 Les conclusions de l'avocat général, conformes à l'analyse de la juridiction de renvoi ${ }^{18}$, ont été ignorées par la Cour de justice qui s'est rangée du côté de la Commission européenne et des gouvernements intervenants ${ }^{19}$. Cependant, si elle a considéré la réglementation italienne comme conforme à la directive retour, c'est en usant d'un raisonnement pour le moins surprenant qu'aucune des parties prenantes ne lui avait soumis.

12 La Commission européenne et les gouvernements intervenants souhaitaient voir la Cour se départir des arrêts El Dridi et Achughbabian au motif que Skerdjan Celaj présentait la spécificité d'être un « récidiviste » de l'irrégularité. Cet argument n'était 
juridiquement pas recevable : comme l'a rappelé l'avocat général, «l'objectif premier de la directive 2008/115 est non pas de prévenir un séjour irrégulier, mais d'y mettre un terme $»^{20}$. Le champ d'application et l'objet de la directive retour sont en effet distincts de ceux des dispositions relatives à l'entrée irrégulière. Or, dans le champ d'application de la directive retour, les États membres sont obligés d'adopter une décision de retour à l'encontre des ressortissants de pays tiers en situation irrégulière (à moins qu'ils ne leur accordent un titre de séjour en vertu de dispositions nationales spécifiques) ${ }^{21}$. Il en résulte qu'indépendamment du nombre de fois où un ressortissant de pays tiers est entré irrégulièrement sur le territoire d'un État membre, ce dernier est toujours tenu de respecter la gradation des mesures coercitives prévues par la directive retour et de ne pas compromettre son effet utile en priorisant la condamnation de l'intéressé à une peine privative de liberté2 ${ }^{2}$.

Bien consciente de l'inconsistance juridique du raisonnement proposé au soutien de la réglementation italienne, la Cour de justice a emprunté une autre voie pour la sauver. Ce faisant, elle a choisi de distordre les arrêts El Dridi et Achughbabian dans ce qui apparaît comme une tentative de cohérence pour le moins ratée. En effet, c'est en rappelant brièvement le principe selon lequel une sanction pénale ne peut intervenir qu'après l'échec d'une procédure de retour qu'elle valide une réglementation prévoyant une sanction pénale applicable avant même l'adoption d'une décision de retour (\$§ 29-30).

La Cour de justice rejoue ici un tour de magie déjà usité par lequel une règle violée dans une procédure $\mathrm{X}$ ne l'est pas réellement du fait de son respect dans une procédure $\mathrm{Y}$. En effet, tout en désignant la procédure de retour dont Skerdjan Celaj avait fait l'objet comme close du fait de son retour autonome vers le territoire d'un État tiers, elle le considère toujours comme ayant fait l'objet d'une procédure de retour infructueuse lorsqu'il est arrêté du fait d'un nouveau séjour irrégulier. Ce faisant, la peine de prison qu'il encourt n'interviendrait ni avant l'adoption d'une décision de retour, ni avant l'épuisement des mesures coercitives prévues par la directive retour, mais bien après l'échec d'une procédure de retour - situation virtuelle matériellement incompatible avec le fait que l'intéressé soit revenu sur le territoire national après l'avoir quitté.

Une fiction juridique similaire avait été utilisée par la Cour de justice s'agissant du droit d'être entendu, qui s'applique à toute procédure susceptible d'aboutir à un acte faisant grief $^{23}$. Liant de manière inquiétante le sort des demandeurs d'asile et des ressortissants de pays tiers en situation irrégulière, elle avait alors retenu que la violation du droit d'être entendu dans le cadre d'une procédure de retour n'en était pas une puisque ce droit avait été reconnu à l'intéressée dans le cadre de l'examen antérieur de sa demande d'asile ${ }^{24}$.

16 En dépit des contorsions juridiques auxquelles la Cour de justice s'est livrée, le constat pragmatique de l'avocat général ne peut qu'être approuvé : "quelle que soit la façon de l'envisager: emprisonner une personne retarde finalement un futur retour $\aleph^{25}$. On peut cependant regretter qu'il n'ait pas fait référence au principe de proportionnalité, que la Cour de justice n'a pas non plus évoqué. Pourtant, tant sa jurisprudence antérieure ${ }^{26}$ que la directive retour ${ }^{27}$ elle-même s'y réfèrent pour imposer aux États membres de respecter la gradation des mesures coercitives prévues par ledit texte. En l'espèce, souligner qu'aucune de ces mesures n'a été adoptée à l'encontre de Skerdjan Celaj avant qu'il ne soit poursuivi n'aurait pas été superflu, la question du respect des droits 
fondamentaux étant a minima aussi importante que celle de la répartition des compétences entre États membres et Union européenne.

En toute hypothèse, la Cour de justice renie ici silencieusement les conséquences de sa jurisprudence antérieure quant à l'articulation de la compétence pénale des États membres avec la directive retour, dont la nécessité d'assurer l'effet utile constituait la clé de voute. Cependant, il serait naïf de croire que les juges de Luxembourg renoncent à promouvoir l'objectif de célérité des retours dans le seul souci de ménager les souverainetés nationales. La raison de ce virage jurisprudentiel, qui fait de facto primer la neutralisation sur l'éloignement, doit plutôt être recherchée dans les difficultés auxquelles se heurtent tant l'Union que les États membres quant à la problématique des «migrants non-éloignables ${ }^{28}$.

\section{$2 \%$ - La neutralisation des migrants en situation irrégulière, objectif apocryphe de la directive retour}

En refusant de tirer les conséquences de sa jurisprudence antérieure, la Cour de justice n'a pas tant rejeté l'argument de l'effet utile per se que remodelé l'objectif de la directive retour d'une manière peu conforme à la lettre du texte. L'abandon de l'éloignement comme priorité apparait ainsi comme un aveu d'échec de la politique de retour de l'Union européenne et de la jurisprudence afférente (A), notamment face à la problématique des migrants non-éloignables. Cependant, si l'arrêt Celaj dénote l'impuissance de la Cour de justice à cet égard, il traduit également la persistance d'un déni aux conséquences ravageuses sur le principe de l'État de droit et le respect des droits fondamentaux (B).

\section{A - L'abandon de l'éloignement comme objectif prioritaire : Un aveu d'échec}

19 La validation d'une réglementation nationale permettant de prioriser une peine privative de liberté par rapport à la mise en œuvre d'une procédure de retour est non seulement contraire à l'objectif officiel de la directive retour, mais également dépourvu de toute logique juridique ${ }^{29}$.

20 En effet, l'objectif officiel de la directive retour est double : l'on peut distinguer un volet "efficacité du retour » et un volet "respect des droits fondamentaux». La Cour de justice invoquait jusqu'alors la nécessité d'assurer l'effet utile de la directive retour au soutien du premier volet, négligeant systématiquement le second. Bien que juridiquement et politiquement contestable, cette pratique jurisprudentielle pouvait éventuellement s'appuyer sur une interprétation téléologique orientée de la directive retour. En revanche, quelle que soit l'interprétation que l'on en fasse, la solution retenue dans l'arrêt Celaj ne trouve aucun fondement juridique dans la directive retour, dont l'effet utile est vainement invoqué par la Cour de justice dans cette affaire. Ce dénouement peut s'expliquer (mais non se justifier) par la poursuite d'un objectif de neutralisation des ressortissants de pays tiers en situation irrégulière, ce qui dénote l'échec de la politique de retour de l'Union européenne.

21 La politique de retour de l'Union européenne est intrinsèquement bancale : d'un côté, la directive retour oblige les États membres à adopter une décision de retour à l'égard 
de tout ressortissant de pays tiers en situation irrégulière, favorise le recours à la rétention à des fins d'éloignement dans le cadre de la procédure de retour et en plafonne la durée maximale; d'un autre côté, elle n'impose pas la délivrance d'un titre de séjour aux ressortissants de pays tiers qui ont fait l'objet d'une procédure de retour infructueuse et n'interdit pas le cumul des procédures de retour. La jurisprudence de la Cour de justice se fait le miroir de cette schizophrénie, rappelant qu'un placement en rétention à des fins d'éloignement ne se justifie que tant qu'il existe une perspective raisonnable que l'éloignement ait lieu dans le délai imparti, mais sans traiter du sort des personnes ainsi remises en liberté ${ }^{30}$.

Relâchés dans une zone de non-droit, les intéressés peuvent alors faire l'objet de poursuites pénales et d'un emprisonnement s'ils se situent sur le territoire d'un des nombreux États membres de l'espace Schengen incriminant l'irrégularitée ${ }^{31}$. Avec l'arrêt Celaj, ils peuvent également l'être lorsque la procédure de retour a bel et bien abouti. Certes, le défendeur avait pénétré sur le territoire italien en violation d'une interdiction d'entrée. Cependant, conformément à la directive retour ici interprétée par la Cour de justice, il devra bel et bien faire l'objet d'une décision de retour, dont l'exécution sera comme souvent très hypothétique. En témoignent tant les données statistiques $^{32}$ que le cas personnel de Skerdjan Celaj, qui avait précédemment quitté le territoire italien de son propre chef après que la procédure de retour qui le visait ait été suspendue pour des raisons matérielles.

Si le tribunal de Florence tire les conséquences de la réponse de la Cour de justice à sa question préjudicielle, le défendeur sera donc emprisonné avant de faire l'objet d'une nouvelle procédure de retour qui, si elle n'aboutit pas, conduira à sa remise en liberté sur le territoire italien. S'il a d'abord fait l'objet d'un placement en rétention à des fins d'éloignement, il pourra alors être à nouveau poursuivi pénalement du fait de son statut migratoire ou faire l'objet d'une nouvelle procédure de retour, le choix de la voie administrative ou pénale restant alors à la discrétion de l'État italien. Il pourra également être ignoré des services administratifs et judiciaires, restant toutefois sans statut légal et donc sans droit, maintenu sous la double épée de Damoclès de l'éloignement et de l'emprisonnement.

Ce vide juridique ne permet pas le retour des ressortissants de pays tiers en situation irrégulière, mais tout au plus leur neutralisation ponctuelle. En plus de mettre à mal le principe de l'État de droit, il conduit à dénier le bénéfice droit à la liberté et à la sûreté aux intéressés, alors même que les États membres de l'espace Schengen sont tenus de le garantir à toutes personnes se trouvant dans leur juridiction ${ }^{33}$. À défaut de solution politique et juridique de niveau européen, les États membres recourent plus ou moins ouvertement à des pratiques liberticides non conformes au droit de l'Union ${ }^{34}$.

Si la réponse à la problématique ces migrants non-éloignables relève en premier lieu des institutions européennes parties à la procédure législative et du Conseil européen, reste que la Cour de justice ne fait pas montre du volontarisme requis par la situation. $\mathrm{Au}$ contraire, l'arrêt Celaj vient adouber une politique du déni, et ce dans un contexte migratoire qui commande une remise à plat de la gestion de l'immigration irrégulière dans le cadre de l'espace Schengen. 


\section{B - L'objectif de neutralisation des migrants non-éloignables : La persistance du déni}

Bien qu'il rompe avec la jurisprudence antérieure de la Cour de justice, l'arrêt Celaj peut néanmoins y être rattaché en un point : la solution retenue favorise le phénomène de crimmigration $^{35}$, soit l'enchevêtrement du droit des étrangers et du droit pénal autour de l'objectif de neutralisation des ressortissants de pays tiers en situation irrégulière. À cet égard, il marque seulement un pas supplémentaire vers ce qui était déjà sous-tendu par la possibilité d'infliger des condamnations pénales non privatives de liberté avant ou parallèlement à l'adoption d'une décision de retour. Or, cette réponse à la problématique des migrants non-éloignables n'en est pas une : intolérable sous l'angle $\mathrm{du}$ respect des droits fondamentaux, la politique du vide juridique est également mortifère sur le plan fonctionnel, la neutralisation recherchée n'étant que fictive à long et moyen-terme.

Tendances parallèles, le développement massif du recours à la rétention à des fins d'éloignement ${ }^{36}$ et la pénalisation croissante de l'irrégularité ont donné naissance à un parcours de privation de liberté potentiellement infini pour les ressortissants de pays tiers en situation irrégulière. Or, matériellement, l'enchaînement des enfermements ne peut constituer une solution viable, de même que remettre en liberté des personnes sans statut légal est socialement insoutenable. À cet égard, il faut noter que «des groupes qui étaient auparavant pris en charge par l'assistance sociale étatique ou par des ceuvres de charité privées, tels que les pauvres, les personnes dépendantes des aides sociales et les toxicomanes sont de plus en plus souvent en contact avec le système pénal ${ }^{37}$. Ainsi, le rejet des migrants-non éloignables dans des «limbes juridiques $»^{38}$ qui les condamnent à la misère n'est qu'une autre voie vers une privation de liberté potentielle. En entérinant la possibilité d'emprisonner un ressortissant de pays tiers pour entrée irrégulière avant l'adoption d'une décision de retour qui devra in fine être adoptée, l'arrêt Celaj participe à cette fuite en avant stérile et dangereuse.

Si l'on peut déplorer que la Cour de justice poursuive une logique aussi liberticide qu'inefficace, reste qu'elle y est presque poussée par la Commission européenne. Ainsi, s'agissant des migrants non-éloignables, le groupe de contact « directive retour » de la direction migration et affaires intérieures préconise la possibilité de "re-detention de la même personne ", soit le cumul des placements en rétention à des fins d'éloignement ${ }^{39}$. Le plafonnement de la durée maximale de rétention, écharpe "droits fondamentaux " de la directive retour ${ }^{40}$, est ainsi frontalement remis en cause.

Au niveau national, l'absence de suivi accessible de la transposition de la directive retour par la Commission européenne rend la connaissance des pratiques difficile. Toutefois, l'exemple extrême de la Grèce est à lui seul éloquent: en 2013, face à l'impossibilité fréquente d'exécuter les décisions de retour, la durée maximale de rétention a tout bonnement été supprimée ${ }^{41}$. Ce constat doit bien sûr être mis en perspective avec le manque de solidarité des États européens en matière de gestion de l'immigration : ceux d'entre eux dont les frontières coïncident avec celles de l'espace Schengen sont en effet placés dans une situation intenable qui, si elle ne légitime pas les violations des droits fondamentaux qu'ils peuvent tolérer ou perpétrer dans leur juridiction, reste un facteur d'explication décisif. 
Les Lettres « Actualités Droits-Libertés » (ADL) du CREDOF (pour s'y abonner) sont accessibles sur le site de la Revue des Droits de l'Homme (RevDH) - Contact

\section{NOTES}

1. CJUE, El Dridi, 28 avril 2011, C-61/11 PPU, §§ 55-59 - ADL du 29 avril 2011.

2. CJUE [GC], Alexandre Achughbabian, 6 décembre 2011, C-329/11, §§ 28, 33, 36-37, 43, 46-49 - ADL du 7 décembre 2011.

3. CJUE, Md Sagor, 6 décembre 2012, C-430/11 , §§ 36, 39-42 et 44-46 - ADL du 12 décembre 2012.

4. Sauf mention contraire, nous entendrons par «États membres » les États soumis à la directive retour, soit les États membres de l'espace Schengen. Il s'agit de l'ensemble des États membres de l'Union européenne à l'exception du Royaume-Uni et de l'Irlande, ainsi que les quatre États membres de l'Association européenne de libre échange (AELE) que sont l'Islande, le Liechtenstein, la Norvège et la Suisse.

5. Conclusions de l'A.G. M. SZPUNAR, présentées le 28 avril 2015, § 4.

6. Sur ce décret législatif, v. P. BONETTI, L'éloignement de l'étranger en situation irrégulière en Italie après la transposition de la directive « retour ", in C. SEVERINO (dir.), La transposition de la directive retour : France, Espagne et Italie, Bruxelles, Bruylant, 2015, pp. 61-104.

7. CJUE, Hassen El Dridi, 28 avril 2011, préc., $\$ 41$; CJUE, M. G. et N. R., 10 septembre 2013, C-383/13 PPU, § 42 .

8. Conclusions de l'A.G. M. SZPUNAR, préc., § $29 \mathrm{~s}$.

9. Id., § 30 .

10. Art. 63 al. 1 pt. 3 b) CE devenu 79 § 2 c) TFUE.

11. C. PEYRONNET, Le recours à la rétention à des fins d'éloignement dans le cadre de la directive retour, mémoire de recherche sous la direction du Professeur M.-L. BASILIEN-GAINCHE, master 2 droit européen des affaires, Lyon III, 2015, pt. 148.

12. Cons. 2 de la directive retour; CJUE, Hassen El Dridi, 28 avril 2011, préc., $\S 31$; CJUE, Mehmet Arslan, 30 mai 2013, C-534/11, § 42 ; CJUE [GC], Thi Ly Pham, 17 juillet 2014, C-474/13, § 20 ; CJUE, Sophie Mukarubega, 5 novembre 2014, préc., § 39 - ADL du 18 novembre 2014 ; CJUE, Z. Zh. et I. O., 11 juin 2015, C- 554/13, § 47. 
13. Sur cette notion, v. Services des études juridiques du Sénat, La double peine, $\mathrm{LC} \mathrm{n}^{\circ} 117$, février 2003.

14. CJUE, Md Sagor, 6 décembre 2012, préc., $\$ 36$.

15. CJUE, Md Sagor, 6 décembre 2012, préc., $\S \S 44-46$.

16. CJUE, Hassen El Dridi, 28 avril 2011, préc., § 55 ; CJUE [GC], Alexandre Achughbabian, 6 décembre 2011, préc., $§ \$ 28,46-49$.

17. Conclusions de l'A.G. M. SZPUNAR, préc., §§ $60 \mathrm{~s}$.

18. Id., §§ 47.-48.

19. Gouvernements italien, tchèque, allemand, grec, norvégien et suisse. V. conclusions de l'A.G.

M. SZPUNAR, préc., § 46.

20. Conclusions de l'A.G. M. SZPUNAR, préc., § 57.

21. Art. $6 \S 4$ de la directive retour.

22. CJUE, Hassen El Dridi, 28 avril 2011, préc. ; CJUE [GC], Alexandre Achughbabian, 6 décembre 2011, préc. ; CJUE, Md Sagor, 6 décembre 2012, préc. ; CJUE (ordonnance), Abdoul Khadre Mbaye, 21 mars 2013, C-522/11, § 28.

23. CJUE, M. G. et N. R., 10 septembre 2013, préc., $§ 32$; CJUE, Sophie Mukarubega, 5 novembre 2014, préc., §§ 42, 45, 49 ; CJUE, Khaled Boudjlida, 11 décembre 2014, C-249/13, §§ 34 et 39.

24. CJUE, Sophie Mukarubega, 5 novembre 2014, préc.; v. M.-L. BASILIEN-GAINCHE, T. RACHO, «Quand le souci d'efficacité de l'éloignement l'emporte sur l'application effective des droits fondamentaux ", in La Revue des droits de l'homme, Actualités DroitsLibertés, mis en ligne le 18 novembre 2014 , § 37.

25. Conclusions de l'A.G. M. SZPUNAR, préc., § 60.

26. CJUE, Hassen El Dridi, 28 avril 2011, préc., §§ 38 et 41 ; CJUE, M. G. et N. R., 10 septembre 2013, préc., $\$ 42$.

27. Cons. 16 et art. 8 § 4 de la directive retour.

28. V. COM(2014) 199 final, préc., pp. 11 et 15.

29. V. mutatis mutandis A. ALIVERTI, «Making people criminal: The role of the criminal law in immigration enforcement ", in Theoretical Criminology. vol. 16, $\mathrm{n}^{\circ}$ 4, novembre 2012.

30. CJCE [GC], Said Shamilovich Kadzoev, 30 novembre 2009, C-357/09 PPU, § 67.

31. V. note 11.

32. C. PEYRONNET, Le recours à la rétention à des fins d'éloignement dans le cadre de la directive retour, mémoire de recherche sous la direction du Professeur M.-L. BASILIEN-GAINCHE, master 2 droit européen des affaires, Lyon III, 2015, pt. 134.

33. Sur la notion de juridiction, V. CEDH, [GC], Hirsi Jamaa et al. c. Italie, 23 février 2012, req. $n^{\circ}$ 27765/09 § 74 ; M. FERNANDEZ, La responsabilité du fait des violations des droits de l'homme dans le cadre des opérations maritimes coordonnées par l'Agence Frontex, mémoire de recherche sous la direction du Professeur C. SANTULLI, master 2 droits de l'homme et droit humanitaire, Paris II, 2012.

34. V. infra.

35. V. notamment J. STUMPF, "The Crimmigration Crisis: Immigrants, Crime, and Sovereign power ", in American University Law Review, vol. 56, n² 2, 2006, pp. 367-419.

36. V. M. GAUTIER, «La rétention en droit communautaire », in O. LECUCQ (dir.), La rétention administrative des étrangers : entre efficacité et protection, Paris, L'Harmattan, coll. Bibliothèques de droit, 2011, pp. 14-31.

37. Notre traduction. A. LEERKES, D. BROEDERS, « A Case of Mixed Motives ? Formal and Informal Functions of Administrative Immigration Detention », British Journal of Criminology, vol. 50, $\mathrm{n}^{\circ} 5$, 2010, p. 834. 
38. M.-L. BASILIEN-GAINCHE, "Immigration Detention under the Return Directive: the CJEU shadowed ligths », in European Journal for Migration and Law, $\mathrm{n}^{\circ}$ 17, 2015, pp. 107-129

39. V. Contact group « return directive ", «Brainstorming on best practices in relation to "nonremovable returnees" ", 21 mars 2013, MIGRAPOL CC Return Dir 50, p. 7.

40. Cette avancée doit pourtant être relativisée du fait que la majorité des États membres prévoyaient une durée maximale de rétention avant l'adoption de la directive retour et ont pour certains profité de sa transposition pour l'augmenter. Ainsi, la durée maximale de rétention moyenne des États membres de l'espace Schengen qui prévoyaient déjà un plafonnement a augmenté de $17 \%$. V. C. PEYRONNET, Le recours à la rétention à des fins d'éloignement dans le cadre de la directive retour, mémoire de recherche sous la direction du Professeur M.-L. BASILIEN-GAINCHE, master 2 droit européen des affaires, Lyon III, 2015, pt. 142.

41. Conseil d'État grec, avis 44/2014, 20 mars 2014. V. D. KOROS, "The unlimited extension of immigrant's detention : Towards governance through uncertainty? ", communication présentée lors de la session commune du Common Study Programme on Criminal Justice and Critical Criminology, Corinthe, 31 octobre 2014.

\section{RÉSUMÉS}

Le 1er octobre 2015, la Cour de justice a rendu une nouvelle décision préjudicielle en interprétation de la directive 2008/115/CE (directive "retour»). Comme l'a souligné l'avocat général Maciej Szpunar, il s'agissait de trancher une affaire aux implications politiques, touchant aux deux domaines régaliens que sont le contrôle du franchissement des frontières et le droit pénal. Bien que la Cour de justice tente de l'inscrire dans la continuité de sa jurisprudence, l'arrêt Celaj s'avère en réalité peu conforme aux principes appliqués précédemment quant à l'articulation de la directive retour avec la compétence pénale des États membres. Ainsi, balayant le principe de coopération loyale pourtant largement invoqué auparavant, elle semble faire fi de l'argument de "l'effet utile " qui fondait ses décisions antérieures. À y bien regarder, elle s'attache ici à remodeler l'objectif de la directive retour: son effet utile n'impliquerait pas tant la facilitation de l'éloignement que la neutralisation des ressortissants de pays tiers en situation irrégulière, dont le retour et la condamnation à des peines privatives de liberté seraient deux variantes interchangeables. Lucides quant à la crispation des souverainetés nationales et timides face à la problématique des migrants non-éloignables, les juges de Luxembourg paraissent naviguer à vue, jonglant entre considérations politiques et principes juridiques au détriment tant de l'effet utile de la directive que des droits fondamentaux.

\section{AUTEUR}

\section{CHLOÉ PEYRONNET}

Doctorante en droit public (EDPL - Université Jean Moulin Lyon 3) 